\title{
Editorial
}

\section{Signal Processing for Applications in Healthcare Systems}

\author{
Pau-Choo Chung, ${ }^{1}$ Chein-I Chang, ${ }^{2}$ Qi Tian, ${ }^{3}$ and Chien-Cheng Lee ${ }^{4}$ \\ ${ }^{1}$ Smart Media and Intelligent Life Excellence (SMILE) Lab, Department of Electrical Engineering, \\ National Cheng Kung University, Tainan 70101, Taiwan \\ ${ }^{2}$ Remote Sensing Signal and Image Processing Laboratory (RSSIPL), Department of Computer Science and Electrical Engineering, \\ University of Maryland Baltimore County (UMBC), 1000 Hilltop Circle, Baltimore, MD 21250, USA \\ ${ }^{3}$ Department of Computer Science, University of Texas at San Antonio, San Antonio, TX 78249-1644, USA \\ ${ }^{4}$ Department of Communications Engineering, Yuan Ze University, 135 Yuan-Tung Road, Chungli 320, Taiwan
}

Correspondence should be addressed to Pau-Choo Chung, pcchung@ee.ncku.edu.tw

Received 4 September 2008; Accepted 4 September 2008

Copyright (C) 2008 Pau-Choo Chung et al. This is an open access article distributed under the Creative Commons Attribution License, which permits unrestricted use, distribution, and reproduction in any medium, provided the original work is properly cited.

\section{THEME AND SCOPE}

The cost of healthcare has been skyrocketing over the past decades. This is mainly due to the rapid growth of aging population. To provide more comfortable and effective healthcare services, a recent trend of healthcare has been directed towards deinstitutionalization, community care, and home care. On the other hand, the technologies for healthcare have run an impressive evolution in signal/image processing, computers, and network communications and have facilitated the development of effective signal/image processing techniques in consumer electronics. Accordingly, the quality of community and home healthcare has been significantly improved and many portable devices have also been developed for a wide variety of applications where signal processing-based software plays a pivotal role in their success. The goal of this special issue is to provide most up-to-date and recent advances of signal/image processing techniques in system and network design of healthcare applications and to serve as a forum and venue for researchers in both academia and industries working in this fascinating and emerging area who share their experiences and findings with the readers. The timely need and demand for this special issue can be witnessed by tremendous responses to the announcement of call for papers, where 37 submissions were received, all of which have been gone through in-depth peer review. While many excellent papers were unfortunately left out, 16 papers selected by guest editors to be published in this special issue that cover a wide variety of healthcare applications ranging from medical signal/image processing to system design and development of hardware devices, each of which can be briefly summarized as follows.

The paper entitled "Using intracardiac vectorcardiographic loop for surface ECG synthesis" by A. Kachenoura et al. describes a supervised machine learning approach to reconstruct the surface of ECG signals from EGM signals that are recorded by implanted devices. The proposed method was applied to reconstruct abnormal heart rhythm and exhibited promising results.

The paper entitled "A minimax mutual information scheme for supervised feature extraction and its application to EEG-based brain-computer interfacing" by $\mathrm{F}$. Oveisi and A. Erfanian proposes a two-dimensional mutual information-based feature extraction approach in the sense that an optimal feature set obtained from the data should have maximum joint data redundancy with target classes. The authors develop a so-called minimax mutual information feature extraction (Minimax MIFX) which maximizes the mutual information between a new feature set and target classes while minimizing the data redundancy. Its performance is then evaluated by EEG signal classification to show if the proposed approach performed better than other feature extraction methods in classification accuracy.

The paper entitled "EEG-based subject- and sessionindependent drowsiness detection: an unsupervised approach" by Nikhil et al. develops an unsupervised subjectand session-independent approach for driver drowsiness detection. It demonstrates that the EEG power in the alpha band (as well as in the theta band) is correlated with changes in the driver's cognitive state with respect to drowsiness. 
Based on this result, a linear combination of deviations of the EEG power in the alpha band and theta band from the respective alert models is used for drowsiness detection.

The paper entitled "nonparametric single-trial EEG feature extraction and classification of driver's cognitive responses" by Chin-Teng Lin et al. investigates the use of electroencephalographic (EEG) signal analysis for classification of the driver's cognitive responses to traffic lights. Three feature extraction methods including nonparametric weighted feature extraction (NWFE), principal component analysis (PCA), linear discriminant analysis (LDA), combined with different classifiers including $\mathrm{k}$ nearest neighbor classification (KNNC), and naive Bayes classifier (NBC) are explored to show that the NWFE with NBC gives the best classification accuracy ranging from $71 \%$ to $77 \%$.

The paper entitled "Independent component analysis for magnetic resonance image analysis" by Yen-Chieh Ouyang et al. addresses two disadvantages of the ICA, random initial conditions, and insufficient number of independent components resulting from multispectral images on one end and a disadvantage of the pure pixel-based classifiers, support vector machine (SVM) and Fisher's linear discriminant analysis (FLDA) over mixed pixels in MR images on the other end. It then develops an approach which combines these disadvantages to make them an advantage. Experimental results demonstrate surprising and significant improvements over either ICA or SVM/FLDA applied alone.

The paper entitled "Coorbit theory, multi- alphamodulation frames and the concept of joint sparsity for medical multichannel data analysis" by Stephan Dahlke et al. presents a signal processing technique that detects and separates signal components such as mMCG, fMCG, or MMG by integrating coorbit theory, multi- $\alpha$-modulation frames, and the concept of joint sparsity measures. An interactive procedure is proposed to deliver individual signal components.

The paper entitled "Application of artificial immune system approach in MRI classification" by Chuin-Mu Wang et al. employs clonal selection algorithm (CSA) of artificial immune systems for classification of brain MR images. This is a new trial that brings an artificial immune concept into pattern selection when applied to medical image classification.

The paper entitled "Microarchitecture of a multicore SoC for data analysis of a lab-on-chip microarray" by G. Kornaros and S. Blionas presents a reconfigurable microarchitecture of a lab-on-chip (LoC) microarray device. The LoC consists of a microfluidics part for sample preparation and hybridization, a microsystem part for electronic detection, and a multicore reconfigurable processing part for data analysis. The proposed architecture is able to process microarray data of various sizes ranging from small sizes of genotyping to large scales of gene expression arrays.

In the paper entitled "Design of a versatile and low cost microvolt level A to D conversion system for use in medical instrumentation applications" by K. M. Williams, and N. Robinson, diverse ambient conditions in various clinical environments place significant stress on sensitive instrumentation, especially in clinical environments. This paper presents a microvolt A to D converter and applies it to portable radiation dosimetry instrumentation, which has been tested under diverse clinical conditions and has shown an improvement in signal resolution over analogue techniques.

The paper entitled "A two-microphone noise reduction system for cochlear implant users with nearby microphones-Part I: signal processing algorithm design and development" by Martin Kompis et al. addresses a real need in the cochlear implant community and presents a twomicrophone noise reduction system for conventional hearing aids. The proposed system is physically small, flexible, and computationally inexpensive so that it provides a potential usage in commercial applications for cochlear implant users. The system is described in a two-paper series with this paper served as the first part on signal processing algorithm design and development and its performance evaluation described in the following paper as the second part of the series.

The paper entitled "A two-microphone noise reduction system for cochlear implant users with nearby microphones_-Part II: performance evaluation” by Martin Kompis et al. is a follow-up of the previous paper on algorithm design and development. It is the second part of a two-paper series on two-microphone noise reduction system which is focused on performance evaluation by simulated environment and physically real anechoic and reverberant environments. The methodology and experimental results will be of interest to the cochlear implant community, the hearing aid community as well as any others who are interested in noise reduction in portable communication systems.

The paper entitled "Hardware implementation of a spline-based genetic algorithm for embedded stereo vision sensor providing real-time visual guidance to the visually impaired" by Dah-Jye Lee et al. develops an embedded stereo vision sensor for visual guidance for people with visual impairment. One-dimensional (1D) spline-based genetic algorithm is applied to matching signals and generating a dense disparity map, from which $3 \mathrm{D}$ information is extracted. The 1D spline-based genetic algorithm can be executed in parallel and implemented into an FPGA to become a compact system.

The paper entitled "Embedded system for real-time digital processing of medical ultrasound Doppler signals" by Stefano Ricci et al. develops an embedded Doppler ultrasound (US) system for real-time processing of digital US signals which are capable of transmitting arbitrary waveforms, simultaneously demodulating the echoes by different frequencies as well as processing the received data through designed programmable algorithms. Since the proposed embedded system is easily programmed, it can be adapted to a wide range of medical applications.

The paper entitled "Computational issues associated with automatic calculation of acute-myocardial-infarction (AMI) scores" by J. B. Destro and S. J. S. Machado explores computational issues in terms of required memory space and computation cost of three-principal AMI scores (Selvester, Aldrich, Anderson-Wilkins) by using digital electrocardiographic (ECG) signals as test examples. It is found that 
the AMI scores can be computed in real time, which makes AMI high potential for urgency applications in telemedicine systems.

The paper entitled "Object delineation by k-connected components" by Paulo Miranda et al. develops an image foresting transform for object delineation based on $\mathrm{k}$ connected components with and without competition among seeds. It provides an application case study in MRI segmentation which will be of interest to researchers working in the field.

The paper entitled "Detect key genes in classification of microarray data" by Yihui Liu addresses detection of key information from high-dimensional microarray profiles using wavelet analysis and genetic algorithm. The wavelet transform is used to extract approximation coefficients, while the genetic algorithm is applied to select features optimized from a gene model reconstructed based on orthogonal approximation coefficients.

$$
\begin{array}{r}
\text { Pau-Choo Chung } \\
\text { Chein-IChang } \\
\text { Qi Tian } \\
\text { Chien-Cheng Lee }
\end{array}
$$

